\title{
Electric Vehicles Operations Oriented Network and Data Analysis
}

\author{
Haohan $\mathrm{Hu}^{1, \mathrm{a}}$, Qiang Luo ${ }^{1, \mathrm{~b}}$ and Jinglin Guo ${ }^{1, \mathrm{c}}$ \\ ${ }^{1}$ Beijing China Power Information Technology Co.,Ltd.,State Grid Electric Power Research \\ Institute,Haidian District,Beijing 100192,China
}

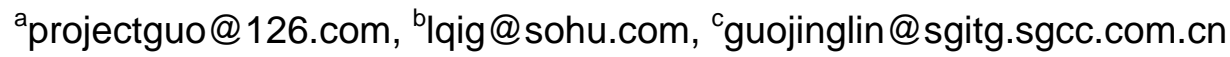

Keywords: Electric vehicles, Interaction network, Operations, Big data analysis

\begin{abstract}
We analyze characteristics of electric vehicles interaction network and present a big data analysis method for electric vehicles operations, which is based on cloud computing model. For the electric vehicles large-scale operations, we propose a scalable architecture design as well as the method to improve the data computing speed.
\end{abstract}

\section{Introduction}

Electric cars are clean and green, thus the development of electric cars is regarded as an effective method of energy saving and emission reduction for traffic system. However, the disadvantages such as inconvenience of battery charging, short range and other factors restrain the popularization of electric vehicles. The large-scale popularization of electronic cars needs country, company and individuals to participate in. Therefore, building a complete electric vehicles operational support system is not only the market target of power grid but also their social responsibility.

\section{Background}

As a new industry, there has been a lot of researches about electric vehicles, but has not yet to establish a mature business model and large-scale operations. The market environment and user scale require cultivation and development by joint efforts of several aspects. The company 'Better Place' which found in 2007 positioned the itself as "provider of global electric vehicle network and service”, and designed networked charging facilities of "battery rental + battery exchange", exploring a new application mode. In 2015, the electric car ownership of China is about 1 million. Besides, China now ranks as first in the world for the number of charging stations and charging piles. Therefore, China has become the country with the greatest potential for electric vehicles operations. With the application of "graphene" technology, electric vehicles will be undoubtedly popularized and gradually begin operating in large-scale in the future. Thus, it is necessary to explore the operational architecture of electric vehicles, which can adapt to the increasing scale of the electric cars.

\section{Interaction network characteristics of electric vehicles}

With the development of automobile electronic technology, software system has been widely used in ordinary cars. The cars have become the "fourth screen" after TV, computer and mobile phone. Navigation and mobile computing technology have been deeply applied to cars. Compared with traditional auto industry, the industry scale of electric cars is smaller. However, the huge potential business opportunities promote the software development of electric vehicles in automobile manufacture industry, which results in gradually developmental Internet of Vehicles.

Compared with the traditional cars, electric cars need more network support. For example, in order to eliminate the driver's worries about the range, drivers need to know the car battery status in time and find convenient charging stations. This demand involves real information data of the charging stations network, and is closely related to the payment system and telecom operators' network. Various applications such as mobile computing and social networks find their natural carrier. For example, value-added services could be expanded into automobile data recorder. Value-added services based on electric vehicles can be regarded as the fusion of telegraphic value-added services 
and electric value-added services. Therefore in the performance form of the social network businesses, there is no significant difference between this field and other fields. They link public through certain ties and are consistent with the characteristics of "small world" network ${ }^{[1,2]}$. Some works discussed about the distribution network planning concerning to the load demand of new electric cars charging. But at the abstract level, this kind of work does not affect the structure characteristics of the electric car operations network.

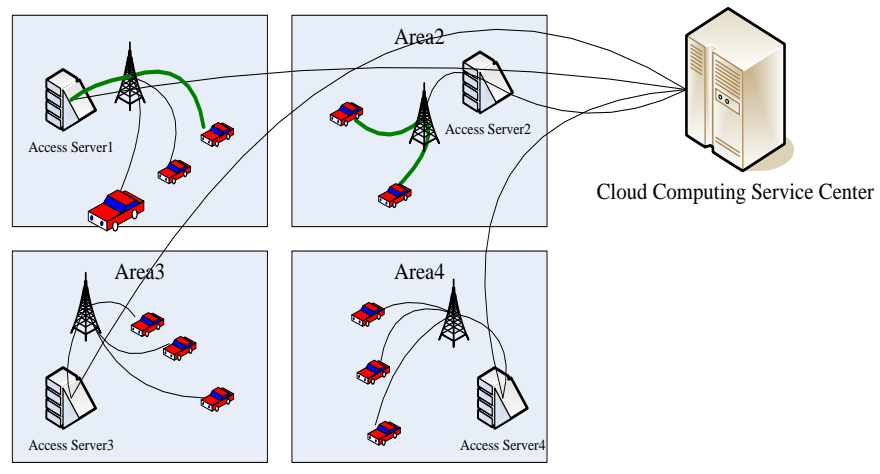

Fig.1: Electric vehicles operations network

On the road, each car could share its local traffic information with existing means of traffic information collection and release channels, thus form the most comprehensive dynamic traffic information. The advantage of such information collected mean is that there is no need to install acquisition points in advance, because a social network is constituted by various kinds of electric cars with their traffic information. With the growing number of service quantity that underlying communication could support, we could build a logic network in application layer, named service overlay network (SON) ${ }^{[3]}$. Comprehensively considering the electric car communication situation under the condition of various network models fusion, communication gateways could be designed for electric cars, which would provide basic support for various business accesses and interactions, thus improving the operating efficiency of electric vehicles, which is consistent with the development direction of future power grid

\section{Data process methods of electric vehicles}

If online operating the electric car in large-scale, the main data of the electric car should include electricity information and traffic information. Because the data collection cycle is second class, big data would be obtained. In the era of big data, data is the key element of continuous operations of the electric car, which need to be properly handled ${ }^{[4,5]}$.

Data characteristics. As mass data of electric cars should take full advantage of power information's regional characteristics, front-end processes are required, including supporting for distributed data compression, data gathering, and data filtering and so on.

According to the real-time characteristic of data, we can divide analysis into two kinds of model, including real-time data analysis and off-line data analysis. In order to analyze data without affecting the user experience, real-time analysis is often required to analyze billions of lines of data in a few seconds. To meet this demand, we could use carefully designed traditional relational databases to constitute parallel processing clusters, or use some memory computing platforms. If the feedback time is not strict, off-line analysis should be applied, which log data into special analysis platforms by data collection tools.

According to the amount of data, we can divide analysis into memory level and massive level. Memory level refers to the data amount that is less than the maximum memory of front-end computing terminal. Actually, even the memory of current PC servers could achieve TB level. We could use some memory databases, which store hotspot data into memory, thus we would get very fast analysis ability and could real-time process front-end business. 
Massive data need to use data center's processing ability. Because of the cost of hardware and software, the universal enterprises adopt HDFS distributed file system of Hadoop to store data, and use MapReduce to analysis.

Dispose of the information in inner network. The electricity information used by electric vehicles is collected by terminal sensors. Using the vast communications network, we can create a real-time, high speed and two-way flow of information, and then ensure the normal operation of all kinds of systems and support all kinds of businesses as usual. Data can be transferred to convergence equipments through a large amount of the underlying collection acquisition and sensing equipment layer together. The larger the size of network is, the greater the data flow is, and "Funnel effect" will be more pressured. In the practical application of smart power, environmental information collected by sensor nodes of similar positions tend to be more similar, and therefore need to apply network polymerization technology.

The collected information has the characteristics in two aspects: on the one hand, the data collected from multiple terminals has different characters and the data type is not the same. Thus, we need to unified data format in the process of fusion at first; On the other hand, a large number of terminal equipments are installed on the outside of the different geographical locations, or in different environments, which will inevitably lead to the information accessed by equipment inaccurate and even may also exist contradictions between information.

Because the time and space of electricity data are sensitive, so the effective integration of time and space changing is necessary. Based on the evidence theory of information fusion method of rough fuzzy set, the basic idea is: first, dealing with the discrete data after the aggregation through the rough set, and then removing redundant data; second, regarding the pretreatment of the observation parameters as separate evidence, and then solving the basic probability assignment through fuzzy membership degree; finally, making a quadratic convergence on the space - time sequence.

Collaborative process. Collaboration and self-organization are important characteristics of a scalable architecture. Considering the architecture of "Internet of things" in the field of intelligent power, the combination mode of cloud computing and sea calculation is conformed to the feature of this field. Multiple computing terminals of sea calculation in systems are involved in collaboration and self-organization. Collaborative approaches can be roughly divided into two categories, centralized and distributed. Centralized coordination means that there is an "omniscient" node in the network, which knows each node's processing tasks, such as resource utilization and the speed of tasks' processing. Besides, this "omniscient" node also collects and monitors the task load, task type and resource using of each node and unified scheduling, undertake the task of all nodes in the system. The scheduling effect of this method is good and can achieve the global optimal, but the scheduling algorithm is complex, communication and computing cost is are large, and often fails to reflect the state change of the node. Especially, it is difficult to achieve when the number of nodes is large. In an open environment, it is extremely difficult to adopt the method of centralized collection to manage node status information and make scheduling decisions. However, the nodes in distributed mechanism are equal and the nodes can decide how to carry out the task scheduling according to their status and the request of the other nodes.

\section{Implantation of data analysis}

Architecture design. Talia proposed that we can offer cloud computing data mining services from four levels ${ }^{[6]}$ : the bottom layer forms the basic steps of data mining algorithm; The second layer is a separate data mining service, such as classification, clustering, etc.; The third layer is distributed data mining models, such as parallel sorting, machine learning of aggregate type, etc.; the fourth layer is data mining applications based on the previous three layers. On the basis of such design, a data mining open service framework based on cloud computing is designed, and a series of data mining service systems have been developed. At the same time, users can define their own workflow through the graphical interface, and then perform it on the platform. 
In this paper, the architecture above forms to the calculating layer. In addition, the whole work also includes the tools of query layer and storage layer, which aims to provide corresponding services for different users.

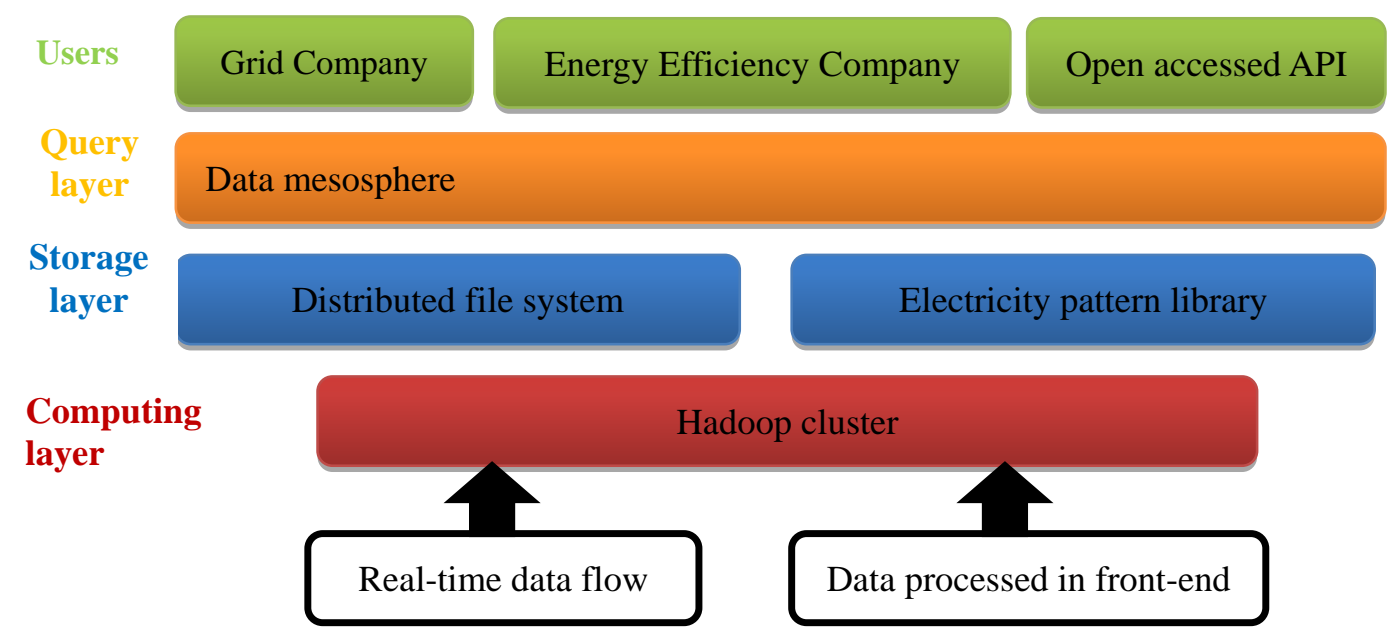

Fig.2 Data mining architecture of electric vehicles

Speed up the data analysis and calculation. Traditional data calculation generally is with a three layers structure, including compute nodes, data nodes and optical fiber network. The advantage of this approach is that it is very popular and easy to work. Users can build Hadoop cluster based on this architecture quickly. But in fact, this kind of structure restricts the performance of Hadoop greatly. In this work, PCIe2.0 communications and FPGA acceleration were adopted. Practice shows that our method calculating speed is about 10 times faster than traditional three-layer structure in the same configuration, data computing node, and under the condition of equal amount of data.

\section{Conclusions}

Increasing haze weather touches every aspect of society greatly, which contributes to the idea of reducing the energy consumption. The electric vehicles are clean and environmental protection, so the development of the electric vehicles is considered as an effective way of energy conservation and emissions reduction in traffic system. Therefore, practical and feasible operating technology will greatly promote the development of electric vehicles.

\section{References}

[1] M. E. J. Newman, Scientific collaboration networks. I. Network construction and fundamental results, Phys. Rev. E64 (2001), 016131

[2] R. Albert, H. Jeong, A.-L. Barabasi. Attack and error tolerance of complex networks. Nature, vol.406.(2000), pp.378-382

[3] Li. Y, Huai JP, Guo HP. A Hierarchical Service Composition Framework Based on Service Overlay Networks. Journal of Software, 18(12),(2007),pp.2967-2979

[4] Hey A J G. The fourth paradigm: data-intensive scientific discovery , (2009)

[5] Howe D, Costanzo M, Fey P, et al. Big data: The future of biocuration . Nature, 455(7209),(2008),pp.47-50.

[6] Domenico Talia: Clouds for Scalable Big Data Analytics. IEEE Computer 46(5), (2013), pp.98-101 